\title{
Afferriamo la questione: cerchiamo sempre di essere ferrati in materia e non commettiamo mai gesti efferati
}

Raffaella Setti

PUBBLICATO: 23 LUGLIO 2019

\section{Quesito:}

Ci sono giunte numerose domande a proposito di quale tra le forme ferrato, afferrato o efferato sia quella giusta da usare nell'espressione essere ferrato/afferrato/efferato in (una) materia nel senso di 'essere preparato, competente, esperto in quella particolare materia'.

Afferriamo la questione: cerchiamo sempre di essere ferrati in materia e non commettiamo mai gesti efferati

I

n molti ci chiedono chiarimenti sulle possibilità di sovrapposizione nell'impiego di ferrato, efferato e afferrato. A partire dai singoli significati e ambiti d'uso, che mostrano quanto i tre aggettivi non offrano margini per parlare di sinonimia, proviamo a ipotizzare i processi linguistici che possono invece indurre alcuni parlanti a sovrapporre e scambiare forme e significati.

Partiamo da ferrato, un aggettivo, dal participio passato del verbo ferrare, a sua volta derivato direttamente da ferro, che pertanto ha come primo significato quello di 'munito di ferro', 'rinforzato con elementi di ferro'. Già presente in alcuni testi pratici toscani del XII sec. ("Gratiolo v staia ad staio ferrato.", Affitti di Coltibuono, riportati nella banca dati dell'OVI e consultabile anche su bibliotecaitaliana.it), ferrato è poi ampiamente attestato dal Trecento (nel GDLI il primo esempio riportato è tratto da Giovanni Villani). Questa prima accezione nasce dalla pratica antica di rinforzare con placche, borchie, lamiere di ferro tutto ciò che doveva resistere all'usura o ad attacchi esterni (quindi scarpe, armature, infissi, ecc.); in particolare la ferratura riguarda i cavalli: un cavallo ferrato è pronto, munito di quello che occorre per essere cavalcato o impiegato in lavori faticosi. A epoche più recenti risalgono la strada ferrata, che cederà poi a ferrovia, e le vie ferrate, quelle alpine, dotate di ferri per facilitarne la scalata. Da questo significato originario si passa a quello di 'duro', 'ostinato', riferibile anche a persone, e poi a 'temprato' quindi 'allenato', 'esercitato', con sporadici esempi a partire dal Settecento che si fanno più frequenti nel corso del Novecento (il GDLI riporta un passo da Roma vestita di nuovo, 1957, di Corrado Alvaro: "Occorre esser ben ferrati per vivere a Roma, riducendo al minimo le esaltazioni, i turbamenti, le prostrazioni”). La Crusca V, in senso figurato e sempre riferibile alla persona, registra invece un altro significato, quello di 'solido, robusto economicamente': "E Figuratam., detto di persona, vale tanto ricco da non sentire gli effetti di qualche danno che patisca ne' propri interessi; ed altresi molto agiato e destro nel procurare il proprio vantaggio". La prima attestazione diferrato nel senso di 'ben preparato, esperto in qualche materia o argomento' (dalla tempra fisica si passa a quella intellettiva) è sempre settecentesca, secondo quanto ci riporta il DELI, che cita Anton Maria Salvini ("di mente assai ferrata". av. I729), mentre la prima attestazione riportata nel GDLI è del 1955 nell'autobiografia di Ardengo Soffici Fine di un mondo ("L'unica materia nella quale avessi poco da apprendere e anzi mi sentissi ormai abbastanza ferrato, era quella dell'arte"), a cui seguono passi tratti dalle prose di Sbarbaro (Scampoli, i960) e di Montale (Auto da fé, I966). Nei dizionari sincroniciferrato, nel significato di 'esperto, ben preparato in un determinato campo', è marcato come familiare (Vocabolario Treccani online, che segnala come molto frequente anche la forma del superlativo ferratissimo), comune (GRADIT) e inserito in esempi da cui si deduce che prevede la 
reggenza della preposizione in (il DISC precisa: "regge la prep. in SIN esperto: essere (molto)f. in qlco.").

Afferrato, participio passato del verbo afferrare, dal latino adferrare 'impugnare il ferro, l'arma', condivide con ferrato la base ferro e in origine aveva il significato di 'impugnato' (riferito al ferro, cioè l'arma), da cui 'stretto saldamente con la mano' che slitta al significato metaforico di 'afferrato col pensiero', quindi 'compreso, capito'. La prima attestazione dell'impiego del verbo in quest'ultima accezione ce la riporta il Tommaseo-Bellini (che attinge a sua volta alle raccolte lessicografiche di Giovanni Gherardini), citando un passo tratto dal Commento di Annibal Caro del I539 al componimento burlesco di Francesco Maria Molza, La Ficheide (noto anche come Capitolo dei fichi del Padre Siceo), di soggetto decisamente licenzioso e per questo escluso dalla lessicografia storica precedente: "Basta solo che voi afferriate il punto, che le fave... sono il principio della generazione". L'espressione che ci interessa, afferrare il punto, rimanda proprio al significato di 'comprendere, cogliere con il pensiero' che ritroviamo nell'italiano contemporaneo in forme del tutto analoghe: oltre allo stesso afferrare il punto, afferrare il nocciolo della questione, il concetto, il senso di qualcosa, ecc. In forma passiva, sempre in senso metaforico, si può essere afferrati da un dubbio, da un rimorso, anche se, in verità, si tratta di espressioni abbastanza rare e di sapore letterario. Niente a che vedere però con ferrato, nel senso di 'esperto', se non attraverso passaggi logici ulteriori e approssimativi: chi ha afferrato ('capito') bene qualcosa può essere ferrato ('esperto') in quell'ambito; in realtà, il concetto di afferrare si riferisce normalmente a qualcosa di puntuale e circoscritto (un punto, un concetto, un significato specifico, ecc.), mentre l'essere ferrato in qualcosa presuppone la conoscenza ampia e approfondita di un argomento o di una disciplina.

Efferato ha tutt'altra origine etimologica: è infatti un latinismo derivato direttamente da efferatu $(m)$, participio passato di efferare 'rendere feroce', composto parasintetico di fera 'fiera, bestia feroce', col prefisso ex-rafforzativo. Presente in italiano già dal XIV secolo, è una voce dotta che ha mantenuto il significato originario di 'feroce, brutale, inumano', oggi utilizzato prevalentemente nella lingua della comunicazione in collocazioni stereotipiche (accostamenti cristallizzati di parole) del tipo crimine efferato, delitto efferato, omicidio efferato, strage efferata, violenza efferata, ecc. Una veloce ricognizione nell'archivio della "Repubblica" (ricerca effettuata l'8/7/2019) rende l'idea della presenza pervasiva dell'aggettivo: efferati 2151/ efferator 846/ efferata 88I/ efferate 534; in collocazione (anche a distanza nel testo) le più frequenti sono omicidio efferato (740); delitto efferato (684); crimine efferato (232); gesto efferato (I82); poi anche violenza efferata (309); strage efferata (II4). Una frequenza d'uso dovuta senz'altro a quella "pigrizia" che porta il linguaggio giornalistico a riutilizzare sempre le stesse formule e che, anche in questo caso, ha come effetto un impiego eccessivo dell'aggettivo, in linea senz'altro con la tendenza all'amplificazione e spettacolarizzazione (soprattutto in negativo) delle notizie; tale "abuso" contribuisce ad "alleggerire" il peso delle parole e a farle percepire come ben conosciute anche nei casi in cui non se ne dominino completamente il significato e la pertinenza ai contesti. Proprio questa martellante presenza nei media ha reso l'aggettivo molto "orecchiato", letto o ascoltato, e pertanto altamente disponibile nella competenza, almeno passiva, dei lettori/ascoltatori. Ma dobbiamo constatare che le incertezze sono sempre in agguato se alcuni interlocutori ci chiedono se sia appropriato l'uso di efferato in sequenze del tipo "è efferato in materia" (come sinonimo di ferrato nel senso visto sopra).

Dopo aver visto origini, significati e usi delle tre forme, proviamo a esaminare nello specifico le ragioni che possono aver generato dubbi e confusione fra ferrato, efferato e afferrato, facendo prima di tutto due precisazioni:

I. In rete le occorrenze che testimoniano questi "scambi" sono decisamente contenute: su Google (ricerca nelle pagine in italiano al 3/7/2019) risultano 54 occorrenze per delitti afferrati (6 
occorrenze per il singolare), 9 per crimini afferrati (4 al singolare), 6 per omicidi afferrati, 3 per stragi afferrate. Un po' più rilevante la presenza di efferato in materia con 699 risultati (740 per il plurale), ma comunque marginale rispetto alle quasi 40.000 occorrenze dell'appropriato ferrato in materia.

2. A proposito della segnalazione fatta da una nostra interlocutrice per cui nel Sabatini-Coletti le due espressioni essere ferrato (in qualcosa) ed essere afferrato sarebbero indicate come sinonimiche, devo dire di non aver trovato riscontro, in questo confortata anche dalla verifica dello stesso Francesco Sabatini: nessuna definizione e nessun esempio riportati nel dizionario suggeriscono un'interpretazione simile.

Per vedere allora che cosa determina la sostituzione tra queste forme, partirei da efferato, parola dotta che, malgrado la sua ampia circolazione nella lingua della comunicazione, rischia di essere mal dominata da molti parlanti. Se alla base c'è una conoscenza solo approssimativa del significato di efferato, si può tendere ad assimilare questa parola di registro alto ad un vocabolo più familiare, magari dalla pronuncia assonante: tecnicamente si parla di malapropismo, un fenomeno che si manifesta in special modo in chi ha difficoltà a dominare i registri più ricercati della lingua. Molto frequenti, ad esempio, le storpiature di termini specialistici medici del tipo migragna ('emicrania'), febbrite ('flebite'), ma anche gli scambi di prefissi e suffissi che portano a affetti personali invece di effetti personali (si veda la risposta di Manuela Manfredini su "La Crusca per voi"), alla sovrapposizione di dorso e torso (si veda la risposta di Vittorio Coletti su "La Crusca per voi"), eccesso invece di accesso di tosse (per questo rimando alla risposta di Francesco Sabatini) o a comprensibile per comprensivo. In generale, vengono applicati in modo inconsapevole meccanismi che portano a ricollocare nell'ambito del noto ciò che non lo è; un passaggio che può interessare anche forestierismi poco praticati (fare il make-up dei dati invece di 'fare il back-up', o entrare con il bengio invece che con il 'badge') e di cui Totò ci ha lasciato memorabili trovate geniali: mo' esce Antonio sciampagna per Moet Chandon Champagne, occhiali da miope e da preside, adire alle vie letali, e molte altre. Come malapropismo, per semplice assonanza, si può spiegare la sostituzione, peraltro non cosi diffusa come abbiamo visto, di efferato con afferrato (uno scambio analogo a quello, ad esempio, tra vertere e versare). Per spiegare lo scambio traferrato ed efferato dobbiamo presupporre che, all'insicurezza nell'uso di efferato, si sommi una parallela scarsa dimestichezza con l'accezione estensiva di ferrato per 'esperto': da questo incerto dominio di entrambe le forme può generarsi la confusione favorita, a mio avviso, anche dalla concrezione della sequenza "é ferrato" (in materia), pronunciata in molte zone d'Italia con raddoppiamento fonosintattico, quindi efferrato che nella pronuncia romanesca (zona da cui proviene una delle domande), ma anche in alcune aree dell'Italia centrale, può diventare efferato con scempiamento della $r$. D'altro canto, può capitare di sentire pronunciare efferrato, usato peró in senso proprio, sempre con raddoppiamento della $r$ : in questo caso si può supporre abbia agito quello che tecnicamente è definito ipercorrettismo, cioè l'intervento volontario del parlante che reintroduce una doppia nella convinzione di ottenere la pronuncia corretta rispetto a quella locale (soprattutto in area romana) che solitamente tende allo scempiamento. Tale raddoppiamento della $r$, certamente erroneo, si riscontra anche nella grafia sporadicamente, ma forse nemmeno tanto, visto che la questione è presente in rete e la troviamo trattata anche in un prontuario per giornalisti curato da Ferruccio Cervi, capo reparto correttori del quotidiano "La Stampa" di lunga esperienza. Talvolta questa grafia, oltre all'ipercorrettismo, può essere dovuta a un raccostamento paretimologico (basato cioè su una etimologia apparentemente plausibile, ma non fondata scientificamente) a ferro e ferrato: si torna cosi a quella tendenza vista prima di ricondurre parole del lessico colto non perfettamente dominate, a parole e forme più comuni e conosciute. Questo efferrato/efferato può, in seguito a una ulteriore storpiatura, trasformarsi in afferrato, anche questa parola decisamente più conosciuta e che con ferrato condivide la base etimologica ferro (afferrare nel senso di 'impugnare il ferro, l'arma'). 
Se queste possono essere le ragioni con cui provare a spiegare gli scambi, peraltro poco rilevanti quantitativamente, fra i tre vocaboli, resta sempre valido il consiglio di accertarsi di conoscere bene significati e usi di ciascuna parola prima di utilizzarla, specialmente in testi scritti che possono diventare oggetto di critica e stigma sociale.

Nota bibliografica:

- Vittorio Coletti, Sui significati di torso e dorso, "La Crusca per voi", 47 (II, 2013), p. I6.

- Rita Fresu, Malapropismo, quel vocabolo deforme, Istituto dell'Enciclopedia Treccani (http://www.treccani.it/magazine/lingua_italiana/speciali/errori/Fresu.html).

- Manuela Manfredini, Risposta su effetti/affetti personali, "La Crusca per voi", 54 (I, 20I7), p. I8.

\section{Cita come:}

Raffaella Setti, Afferriamo la questione: cerchiamo sempre di essere ferrati in materia e non commettiamo mai gesti efferati , "Italiano digitale", 2019, X, 2019/3 (luglio-settembre) DOI: $10.35948 / 2532-9006 / 2020.3197$ 